\title{
Leptonic CP Violation and the Baryon Asymmetry of the Universe
}

\section{S. T. Petcov*}

SISSA/INFN, Trieste, Italy, and Kavli IPMU, University of Tokyo (WPI), Tokyo, Japan

\begin{abstract}
The phenomenology of 3-neutrino mixing, the current status of our knowledge about the 3neutrino mixing parameters, including the absolute neutrino mass scale, and of the Dirac and Majorana $\mathrm{CP}$ violation in the lepton sector, are summarised. The seesaw mechanism of neutrino mass generation and the related leptogenesis scenario of generation of the baryon asymmetry of the Universe are briefly discussed. The results showing that the CP violation necessary for the generation of the baryon asymmetry of the Universe in leptogenesis can be due exclusively to the Dirac and/or Majorana CP-violating phase(s) in the neutrino mixing matrix $U$ are reviewed.
\end{abstract}

2nd World Summit: Exploring the Dark Side of the Universe 25-29 June, 2018 - EDSU2018

University of Antilles, Pointe-à-Pitre, Guadeloupe, France

${ }^{*}$ Speaker. 


\section{Introduction: the Three Neutrino Mixing}

The discovery of neutrino oscillations $[1,2,3]$ - transitions in flight between the different flavour neutrinos $v_{e}, v_{\mu}, v_{\tau}$ (antineutrinos $\bar{v}_{e}, \bar{v}_{\mu}, \bar{v}_{\tau}$ ) caused by non-zero neutrino masses and neutrino mixing - opened up a new field of research in elementary particle physics. In the 20 years after the first compelling evidence for oscillations of atmospheric muon neutrinos and antineutrinos $v_{\mu}$ and $\bar{v}_{\mu}$ was provided by the Super Kamiokande experiment in 1998 [4], oscillation of the solar $v_{e}$ neutrinos, accelerator $v_{\mu}$ and $\bar{v}_{\mu}$ and reactor $\bar{v}_{e}$ neutrinos were also observed and the neutrino oscillation phenomenon was extensively studied (see, e.g., [5]). In this period a remarkable progress has been made in the measurement of the parameters which drive the oscillations.

All well understood and compelling neutrino oscillation data can be described within the reference scheme of 3-neutrino mixing in vacuum, which we will consider in the present article:

$$
\mathscr{L}_{\mathrm{CC}}=-\frac{g}{\sqrt{2}} \sum_{l=e, \mu, \tau} \overline{l_{L}}(x) \gamma_{\alpha} v_{l L}(x) W^{\alpha \dagger}(x)+\text { h.c. }, v_{l \mathrm{~L}}(x)=\sum_{j=1}^{3} U_{l j} v_{j \mathrm{~L}}(x) .
$$

Here $\mathscr{L}_{\mathrm{CC}}$ is the leptonic part of the charged current weak interaction Lagrangian, $v_{l L}(x)$ are the left-handed (LH) fields of flavour neutrinos, $v_{j \mathrm{~L}}(x)$ is the LH field of the neutrino $v_{j}$ having a mass $m_{j}$, and $U$ is a unitary matrix - the Pontecorvo, Maki, Nakagawa, Sakata (PMNS) neutrino mixing matrix [1, 2, 3], $U \equiv U_{\text {PMNS }}$. The PMNS matrix, as is well known, can be parametrised in the case of 3-neutrino mixing by 3 angles and, depending on whether the massive neutrinos $v_{j}$ are Dirac or Majorana particles, by one Dirac, or one Dirac and two Majorana, CP violation (CPV) phases [6]:

$$
U=V P, \quad P=\operatorname{diag}\left(1, e^{i \frac{\alpha_{21}}{2}}, e^{i \frac{\alpha_{31}}{2}}\right),
$$

where $\alpha_{21,31}$ are the two Majorana CPV phases. In the "standard" parametrisation [5] the matrix $V$ has the form:

$$
V=\left(\begin{array}{ccc}
c_{12} c_{13} & s_{12} c_{13} & s_{13} e^{-i \delta} \\
-s_{12} c_{23}-c_{12} s_{23} s_{13} e^{i \delta} & c_{12} c_{23}-s_{12} s_{23} s_{13} e^{i \delta} & s_{23} c_{13} \\
s_{12} s_{23}-c_{12} c_{23} s_{13} e^{i \delta} & -c_{12} s_{23}-s_{12} c_{23} s_{13} e^{i \delta} & c_{23} c_{13}
\end{array}\right) .
$$

In eq. (1.3), $c_{i j}=\cos \theta_{i j}, s_{i j}=\sin \theta_{i j}$, the angles $\theta_{i j}=[0, \pi / 2]$, and $\delta=[0,2 \pi)$ is the Dirac CPV phase. It follows from the current data that the three massive neutrinos $v_{1,2,3}$ should have masses not exceeding approximately $0.5 \mathrm{eV}, m_{1,2,3} \lesssim 0.5 \mathrm{eV}$. On the basis of the existing neutrino data it is impossible to determine whether the massive neutrinos $v_{j}$ are Dirac or Majorana fermions.

In the case of 3-neutrino mixing, oscillations involving all flavour neutrinos $v_{l}$ (antineutrinos $\left.\bar{v}_{l}\right), v_{l} \leftrightarrow v_{l^{\prime}}\left(\bar{v}_{l} \leftrightarrow \bar{v}_{l^{\prime}}\right), l, l^{\prime}=e, \mu, \tau$, are possible. The 3-neutrino oscillation probabilities $P\left(v_{l} \rightarrow\right.$ $\left.v_{l^{\prime}}\right)$ and $P\left(\bar{v}_{l} \rightarrow \bar{v}_{l^{\prime}}\right)$ are functions of the neutrino energy, $E$, the source-detector distance $L$, of the elements of $U$ and, for relativistic neutrinos used in all neutrino experiments performed so far, of the two independent neutrino mass squared differences $\Delta m_{21}^{2} \neq 0$ and $\Delta m_{31}^{2} \neq 0,\left(\Delta m_{j k}^{2} \equiv m_{j}^{2}-m_{k}^{2}\right)$ present in the case of 3-neutrino mixing (see, e.g., ref. [7]). In the widely used convention of numbering the neutrinos with definite mass $v_{j}$ we are going to employ, $\theta_{12}, \Delta m_{\odot}^{2}=\Delta m_{21}^{2}>0$, and $\theta_{23}, \Delta m_{\mathrm{atm}}^{2}=\Delta m_{31(23)}^{2}$, represent the parameters which drive the solar $\left(v_{e}\right)$ and the dominant 


\begin{tabular}{lccc}
\hline Parameter & Best fit value & $2 \sigma$ range & $3 \sigma$ range \\
\hline$\Delta m_{31}^{2} / 10^{-3} \mathrm{eV}^{2}(\mathrm{NO})$ & 2.49 & $2.43-2.56$ & $2.39-2.59$ \\
$\left(-\Delta m_{32}^{2}\right) / 10^{-3} \mathrm{eV}^{2}(\mathrm{IO})$ & 2.48 & $2.41-2.54$ & $2.38-2.58$ \\
$\Delta m_{21}^{2} / 10^{-5} \mathrm{eV}^{2}(\mathrm{NO}, \mathrm{IO})$ & 7.34 & $7.05-7.69$ & $6.92-7.91$ \\
\hline $\sin ^{2} \theta_{12} / 10^{-1}(\mathrm{NO})$ & 3.04 & $2.78-3.32$ & $2.65-3.46$ \\
$\sin ^{2} \theta_{12} / 10^{-1}(\mathrm{IO})$ & 3.03 & $2.77-3.31$ & $2.64-3.45$ \\
$\sin ^{2} \theta_{13} / 10^{-2}(\mathrm{NO})$ & 2.14 & $1.98-2.31$ & $1.90-2.39$ \\
$\sin ^{2} \theta_{13} / 10^{-2}(\mathrm{IO})$ & 2.18 & $2.02-2.35$ & $1.95-2.43$ \\
$\sin ^{2} \theta_{23} / 10^{-1}(\mathrm{NO})$ & 5.51 & $4.48-5.88$ & $4.30-6.02$ \\
$\sin ^{2} \theta_{23} / 10^{-1}(\mathrm{IO})$ & 5.57 & $4.86-5.89$ & $4.44-6.03$ \\
$\delta / \pi(\mathrm{NO})$ & 1.32 & $0.98-1.79$ & $0.83-1.99$ \\
$\delta / \pi(\mathrm{IO})$ & 1.52 & $1.22-1.79$ & $1.07-1.92$ \\
\hline
\end{tabular}

Table 1: The best fit values, $2 \sigma$ and $3 \sigma$ ranges of the neutrino oscillation parameters obtained in the global analysis of the neutrino oscillation data performed in [11]. See text for further details. (The Table is taken from ref. [14]).

atmospheric $v_{\mu}$ and $\bar{v}_{\mu}$ oscillations, respectively, and $\theta_{13}$ is associated with the oscillations of reactor $\bar{v}_{e}$ observed in the Daya Bay, RENO and Double Chooz experiments $[8,9,10]$.

The existing data, accumulated over many years of studies of neutrino oscillations, allow us to determine $\Delta m_{21}^{2}, \theta_{12}$, and $\left|\Delta m_{31(32)}^{2}\right|, \theta_{23}$ and $\theta_{13}$, with an impressively high precision [11, 12]. Since 2013 there are also persistent hints that the Dirac CPV phase $\delta$ has a value close to $3 \pi / 2$ (see [13]). We note that the currently available neutrino oscillation data do not allow to fix the sign of $\Delta m_{31(32)}^{2}$. In the 3-neutrino mixing scheme under discussion, the two possible signs of $\Delta m_{31(32)}^{2}$ correspond to two types of neutrino mass spectrum: $\Delta m_{31(32)}^{2}>0-$ to spectrum with normal ordering (NO), and $\Delta m_{31(32)}^{2}<0$ - to spectrum with inverted ordering (IO) (see further).

The best fit values (b.f.v.) and the $2 \sigma$ and $3 \sigma$ allowed ranges of $\Delta m_{21}^{2}, s_{12}^{2},\left|\Delta m_{31(32)}^{2}\right|, s_{23}^{2}, s_{13}^{2}$ and $\delta$ for the NO and IO spectra, found in the latest analysis of global neutrino oscillation data performed in [11] are given in Table 1. The results quoted in Table 1 imply, in particular, that $\Delta m_{21}^{2} /\left|\Delta m_{31(32)}^{2}\right| \cong 0.03$. Thus, we have $\left|\Delta m_{31}^{2}\right|=\left|\Delta m_{32}^{2}-\Delta m_{21}^{2}\right| \cong\left|\Delta m_{32}^{2}\right|$.

It follows from Table 1 that the best fit value of $\theta_{23}$ is somewhat larger than $\pi / 4$, but the value $\pi / 4$ lies within $(1.0-1.5) \sigma$ from the best fit value. The value of $\theta_{12}=\pi / 4$, i.e., maximal solar neutrino mixing, is definitely ruled out by the data. Therefore, one has $\theta_{12}<\pi / 4$ and at $99.73 \%$ C.L., $\cos 2 \theta_{12} \geq 0.30$.

The quoted results imply also that the value of $\theta_{23}$ can deviate by approximately \pm 0.1 from $\pi / 4, \theta_{12} \cong \pi / 5.4$ and that $\theta_{13} \cong \pi / 20$. Thus, the pattern of neutrino mixing differs drastically from the pattern of quark mixing.

In what concerns the Dirac CPV phase $\delta$, the authors of the analysis performed in [11] find that the best fit value of $\delta$ is close to $3 \pi / 2$. More specifically, for NO (IO) spectrum it reads $\delta=$ 
$1.38 \pi(1.52 \pi)$. According to [11] the CP conserving value $\delta=0(2 \pi)$ is disfavored at $3.0(3.6) \sigma$, while the second CP conserving value $\delta=\pi$ is disfavored at 1.8 (3.6) $\sigma$. The $\mathrm{CP}$ violating value $\delta=\pi / 2$ is strongly disfavored at $4.4(5.2) \sigma$ by the data. Finally, at $3 \sigma, \delta / \pi$ is found in [11] to lie in the interval $0.83-1.99(1.07$ 1.93). Thus, we have an indication from the data, although rather weak, for $\mathrm{CP}$ violation in lepton sector due to the Dirac phase $\delta$ (i.e., for leptonic Dirac $\mathrm{CP}$ violation). Very similar results were obtained in the independent global analysis performed in ref. [12].

As we have indicted earlier, the currently available data do not allow to fix the sign of $\Delta m_{31(32)}^{2}$ and the two possible signs of $\Delta m_{31(32)}^{2}$ correspond to two types of neutrino mass spectrum - with normal ordering and with inverted ordering. More specifically, in the convention of numbering of the 3 neutrinos $v_{j}$ with definite mass employed by us the two spectra read:

i) spectrum with normal ordering (NO): $m_{1}<m_{2}<m_{3}, \Delta m_{31(32)}^{2}>0, \Delta m_{21}^{2}>0, m_{2(3)}=\left(m_{1}^{2}+\right.$ $\left.\Delta m_{21(31)}^{2}\right)^{\frac{1}{2}}$;

ii) spectrum with inverted ordering (IO): $m_{3}<m_{1}<m_{2}, \Delta m_{32(31)}^{2}<0, \Delta m_{21}^{2}>0, m_{2}=\left(m_{3}^{2}+\right.$ $\left.\Delta m_{23}^{2}\right)^{\frac{1}{2}}, m_{1}=\left(m_{3}^{2}+\Delta m_{23}^{2}-\Delta m_{21}^{2}\right)^{\frac{1}{2}}$.

Depending on the values of the lightest neutrino mass, $\min \left(m_{j}\right)$, the neutrino mass spectrum can also be:

a) Normal Hierarchical $(N H): m_{1} \ll m_{2}<m_{3}, m_{2} \cong\left(\Delta m_{21}^{2}\right)^{\frac{1}{2}} \cong 8.6 \times 10^{-3} \mathrm{eV}, m_{3} \cong\left(\Delta m_{31}^{2}\right)^{\frac{1}{2}} \cong$ $0.0499 \mathrm{eV}$; or

b) Inverted Hierarchical (IH): $m_{3} \ll m_{1}<m_{2}, m_{1,2} \cong\left|\Delta m_{32}^{2}\right|^{\frac{1}{2}} \cong 0.0498 \mathrm{eV}$; or

c) Quasi-Degenerate $(Q D): m_{1} \cong m_{2} \cong m_{3} \cong m_{0}, m_{j}^{2} \gg\left|\Delta m_{31(32)}^{2}\right|, m_{0} \gtrsim 0.10 \mathrm{eV}$.

Determining the type of neutrino mass spectrum is one of the main goals of the future experiments in the field of neutrino physics ${ }^{1}$ (see, e.g., refs. [5, 15]). According to [11], the absolute $\chi^{2}$ minimum obtained in the analysis of the global neutrino oscillation data takes place for $\Delta m_{31(32)}^{2}>0$, i.e., for NO neutrino mass spectrum, the local minimum in the case of IO spectrum $\left(\Delta m_{31(32)}^{2}<0\right)$ being approximately by $3.1 \sigma$ higher. Thus, according to [11], the existing data favor the NO spectrum over the IO spectrum at approximately $3.1 \sigma$.

All types of 3-neutrino mass spectrum considered above are compatible with the existing experimental upper limits on the absolute scale of neutrino masses. Information about the absolute neutrino mass scale (or about $\min \left(m_{j}\right)$ ) can be obtained, e.g., by measuring the spectrum of electrons near the end point in ${ }^{3} \mathrm{H} \beta$-decay experiments $[17,18,19]$ and from cosmological and astrophysical data. The most stringent upper bounds on the $\bar{v}_{e}$ mass were obtained in the Troitzk [20] experiment:

$$
m_{\bar{v}_{e}}<2.05 \mathrm{eV} \text { at } 95 \% \text { C.L. }
$$

Similar result was obtained in the Mainz experiment[18] : $m_{\bar{v}_{e}}<2.3 \mathrm{eV}$ at $95 \% \mathrm{CL}$. We have $m_{\bar{v}_{e}} \cong m_{1,2,3}$ in the case of QD spectrum. The KATRIN experiment [21], which was commissioned on June 11, 2018, is planned to reach sensitivity of $m_{\bar{v}_{e}} \sim 0.20 \mathrm{eV}$, i.e., it will probe the region of the QD spectrum.

\footnotetext{
${ }^{1}$ For a review of the experiments which can provide data on the type of neutrino mass spectrum see, e.g., ref. [15]; for some specific proposals see, e.g., ref. [16].
} 
Constraints on the sum of the neutrino masses can be obtained from cosmological and astrophysical data (see, e.g., ref. [22]). Depending on the model complexity and the input data used one typically obtains [22]: $\sum_{j} m_{j} \lesssim(0.3-1.3) \mathrm{eV}, 95 \%$ C.L. Assuming the existence of three light massive neutrinos and the validity of the $\Lambda \mathrm{CDM}$ (Cold Dark Matter) model, and using their data on the $\mathrm{CMB}$ temperature power spectrum anisotropies, polarisation, on gravitational lensing effects and the low $l$ CMB polarization spectrum data (the "low P" data), etc. the Planck Collaboration reported an updated upper limit on the sum of the neutrino masses [23], which, depending on the data-set used, varies in the interval: $\sum_{j} m_{j}<(0.340-0.72) \mathrm{eV}, 95 \%$ C.L. Adding data on Baryon Acoustic Oscillations (BAO) lowers the limit to [23]:

$$
\sum_{j} m_{j}<0.170 \mathrm{eV}, \quad 95 \% \text { C.L. }
$$

One should note that the Planck collaboration analysis is based on the $\Lambda$ CDM cosmological model. The quoted bounds may not apply in nonstandard cosmological scenarios (see, e.g., [24]).

Apart from the hint that the Dirac phase $\delta \sim 3 \pi / 2$, no other experimental information on the Dirac and Majorana CPV phases in the neutrino mixing matrix is available at present. Thus, the status of CP symmetry in the lepton sector is essentially undetermined. With $\theta_{13} \cong 0.15 \neq 0$, the Dirac phase $\delta$ can generate CP violating effects in neutrino oscillations $[6,25]$, i.e, a difference between the probabilities of the $v_{l} \rightarrow v_{l^{\prime}}$ and $\bar{v}_{l} \rightarrow \bar{v}_{l^{\prime}}$ oscillations, $l \neq l^{\prime}=e, \mu, \tau$. The magnitude of CP violation in $v_{l} \rightarrow v_{l^{\prime}}$ and $\bar{v}_{l} \rightarrow \bar{v}_{l^{\prime}}$ oscillations, $l \neq l^{\prime}=e, \mu, \tau$, is determined by [26] the rephasing invariant $J_{C P}$, associated with the Dirac CPV phase in $U$ :

$$
J_{\mathrm{CP}}=\operatorname{Im}\left(U_{\mu 3} U_{e 3}^{*} U_{e 2} U_{\mu 2}^{*}\right) .
$$

It is analogous to the rephasing invariant associated with the Dirac CPV phase in the CKM quark mixing matrix [27]. In the standard parametrisation of the neutrino mixing matrix (1.3), $J_{\mathrm{CP}}$ has the form:

$$
J_{C P} \equiv \operatorname{Im}\left(U_{\mu 3} U_{e 3}^{*} U_{e 2} U_{\mu 2}^{*}\right)=\frac{1}{8} \cos \theta_{13} \sin 2 \theta_{12} \sin 2 \theta_{23} \sin 2 \theta_{13} \sin \delta .
$$

Thus, given the fact that $\sin 2 \theta_{12}, \sin 2 \theta_{23}$ and $\sin 2 \theta_{13}$ have been determined experimentally with a relatively high precision, the size of $\mathrm{CP}$ violation effects in neutrino oscillations depends essentially only on the magnitude of the currently not well determined value of the Dirac phase $\delta$. The current data implies $0.029(0.030)|\sin \delta| \lesssim\left|J_{C P}\right| \lesssim 0.035|\sin \delta|$, where we have used the $3 \sigma$ ranges of $\sin ^{2} \theta_{12}, \sin ^{2} \theta_{23}$ and $\sin ^{2} \theta_{13}$ given in Table 1. For the current best fit values of $\sin ^{2} \theta_{12}, \sin ^{2} \theta_{23}$, $\sin ^{2} \theta_{13}$ and $\delta$ we find in the case of NO (IO) spectrum: $J_{C P} \cong 0.033 \sin \delta \cong-0.028\left(J_{C P} \cong\right.$ $0.033 \sin \delta \cong-0.033$ ). Thus, if the indication that $\delta$ has a value close to $3 \pi / 2$ is confirmed by future more precise data, i) the $J_{C P}$ factor in the lepton sector would be approximately by 3 orders of magnitude larger in absolute value than the corresponding $J_{C P}$ factor in the quark sector, and ii) the $\mathrm{CP}$ violation effects in neutrino oscillations would be relatively large and observable.

If the neutrinos with definite masses $v_{i}, i=1,2,3$, are Majorana particles, the 3-neutrino mixing matrix contains two additional Majorana CPV phases [6]. However, the flavour neutrino oscillation probabilities $P\left(v_{l} \rightarrow v_{l^{\prime}}\right)$ and $P\left(\bar{v}_{l} \rightarrow \bar{v}_{l^{\prime}}\right), l, l^{\prime}=e, \mu, \tau$, do not depend on the Majorana phases $[6,28]$. The Majorana phases can play important role, e.g, in $|\Delta L|=2$ processes like 
neutrinoless double beta $\left((\beta \beta)_{0 v^{-}}\right)$decay $(A, Z) \rightarrow(A, Z+2)+e^{-}+e^{-}, L$ being the total lepton charge, in which the Majorana nature of massive neutrinos $v_{i}$ manifests itself (see, e.g, refs. [7, 29, 30]).

Our interest in the CPV phases present in the neutrino mixing matrix is stimulated also by the intriguing possibility that the Dirac phase and/or the Majorana phases in $U_{\mathrm{PMNS}}$ can provide the CP violation necessary for the generation of the observed baryon asymmetry of the Universe (BAU) [31, 32, 34] (see also [35]; for specific models in which this possibility is realised see, e.g., [36]).

Determining the status of CP symmetry in the lepton sector is one of the principal goals of the program of current and future research in neutrino physics. It is part of a very ambitious program of research in neutrino physics, which extends beyond 2030, and which include also [5]:

i) determination of the status of lepton charge conservation and the nature - Dirac or Majorana of massive neutrinos (which is one of the most challenging and pressing problems in present day elementary particle physics);

ii) determination of the spectrum neutrino masses possess, or neutrino mass ordering;

iii) determination of the absolute neutrino mass scale, or $\min \left(m_{j}\right)$.

A successful realisation of this program ${ }^{2}$ is of fundamental importance for making progress in understanding the origin of neutrino masses and mixing and its possible relation to new beyond the Standard Model (BSM) physics.

Understanding the origin of the patterns of neutrino mixing and of neutrino mass squared differences, revealed by the data obtained in the neutrino oscillation experiments is one of the most challenging problems in neutrino physics. It is part of the more general fundamental problem in particle physics of understanding the origins of flavour, i.e., of the patterns of quark, charged lepton and neutrino masses, and of the quark and lepton mixing.

\section{The See-Saw Mechanism, Leptonic CP Violation and Leptogenesis}

It follows from the data discussed in the preceding Section that neutrino masses are much smaller than the masses of charged leptons and quarks. If we take as an indicative upper limit $m_{j} \lesssim 0.5 \mathrm{eV}$, we have $m_{j} / m_{l, q} \lesssim 10^{-6}$, where $m_{l}$ and $m_{q}$ are the charged lepton and quark masses, $l=e, \mu, \tau, q=d, s, b, u, c, t$. It is natural to suppose that the remarkable smallness of neutrino masses is related to the existence of a new fundamental mass scale in particle physics, and thus to new physics beyond that predicted by the Standard Theory.

A natural explanation of the smallness of neutrino masses is provided by the see-saw mechanism of neutrino mass generation [37]. An integral part of the simplest version of this mechanism - the so-called "type I see-saw", are the $S U(2)_{L}$ singlet RH neutrinos $v_{l R}, l=e, \mu, \tau$. The latter are assumed to possess a Majorana mass term as well as Yukawa type coupling with the Standard Theory lepton and Higgs doublets $\psi_{l L}(x)$ and $\Phi(x)$, respectively, $\left(\psi_{l L}(x)\right)^{T}=\left(v_{l L}^{T}(x) l_{L}^{T}(x)\right)$, $l=e, \mu, \tau,(\Phi(x))^{T}=\left(\Phi^{(0)} \Phi^{(-)}\right)$. The Standard Theory admits such a minimal extension which does not modify any of the basic attractive features of the Theory (unitarity, renormalisability, etc.). In the basis in which the Majorana mass matrix of RH neutrinos is diagonal we have:

$$
\mathscr{L}_{\mathrm{Y}, \mathrm{M}}(x)=-\left(\lambda_{k l} \overline{N_{k R}}(x) \Phi^{\dagger}(x) \psi_{l L}(x)+\text { h.c. }\right)-\frac{1}{2} M_{k} \overline{N_{k}}(x) N_{k}(x),
$$

\footnotetext{
${ }^{2}$ See, e.g., [5] for a rather detailed list of current and planned experiments that are foreseen to contribute to the comprehensive long-term program of research in neutrino physics.
} 
where $\lambda_{l k}$ is the matrix of neutrino Yukawa couplings and $N_{k}(x)$ is the heavy (RH) Majorana neutrino field possessing a mass $M_{k}>0, M_{1}<M_{2}<M_{3}$. The fields $N_{k}(x)$ satisfy the Majorana condition $C{\overline{N_{k}}}^{T}(x)=\xi_{k} N_{k}(x)$, where $C$ is the charge conjugation matrix and $\xi_{k}$ is a phase. When the electroweak symmetry is broken spontaneously, the neutral component of the Higgs doublet field develops non-zero vacuum expectation value $v=174 \mathrm{GeV}$ and the neutrino Yukawa coupling generates a neutrino Dirac mass term: $m_{k l}^{D} \overline{N_{k R}}(x) v_{l L}(x)+$ h.c., with $m^{D}=v \lambda$. In the case when the elements of $m^{D}$ are much smaller than $M_{k},\left|m_{j l}^{D}\right| \ll M_{k}, j, k=1,2,3, l=e, \mu, \tau$, the interplay between the Dirac mass term and the Majorana mass term of the heavy singlets $N_{k}$ generates an effective Majorana mass (term) for the LH flavour neutrino fields $v_{l L}(x)$ [37]:

$$
\left(m^{v}\right)_{l^{\prime} l} \cong v^{2}\left(\lambda^{T} M^{-1} \lambda\right)_{l^{\prime} l}=\left(\left(m^{D}\right)^{T} M^{-1} m^{D}\right)_{l^{\prime} l}=\left(U^{*} m U^{\dagger}\right)_{l^{\prime} l},
$$

where $M \equiv \operatorname{Diag}\left(M_{1}, M_{2}, M_{3}\right)\left(M_{1,2,3}>0\right), m \equiv \operatorname{Diag}\left(m_{1}, m_{2}, m_{3}\right), m_{j} \geq 0$ being the mass of the light Majorana neutrino $v_{j}$, and $U$ is the PMNS matrix The diagonalisation of the mass matrix $m^{v}$ leads to the appearance of the PMNS neutrino mixing matrix in the charged current weak interaction Lagrangian $\mathscr{L}_{\mathrm{CC}}(x)$, eq. (1.1).

In grand unified theories, $m^{D}$ is typically of the order of the charged fermion masses. In $S O(10)$ theories [37], for instance, $m^{D}$ coincides with the up-quark mass matrix. Taking indicatively $m^{v} \sim$ $0.05 \mathrm{eV}, m^{D} \sim 100 \mathrm{GeV}$, one finds $M_{k} \sim 2 \times 10^{14} \mathrm{GeV}$, which is close to the scale of unification of electroweak and strong interactions, $M_{G U T} \cong 2 \times 10^{16} \mathrm{GeV}$. In GUT theories with RH neutrinos one finds that indeed the heavy singlets $N_{k}$ naturally obtain masses which are by few to several orders of magnitude smaller than $M_{G U T}$ (see, e.g., ref. [38]).

One of the characteristic predictions of the see-saw mechanism is that both the light and heavy neutrinos $v_{j}$ and $N_{k}$ are Majorana particles. The Majorana nature of the light neutrinos can be revealed in the $(\beta \beta)_{0 v}$-decay experiments (see, e.g., $[5,7,29,30]$

We will discuss next briefly the interesting possibility $[31,32,34]$ that the $\mathrm{CP}$ violation necessary for the generation of the baryon asymmetry of the Universe, $Y_{B}$, in the leptogenesis scenario can be due exclusively to the Dirac and/or Majorana CPV phases in the PMNS matrix, and thus can be directly related to the low energy leptonic $\mathrm{CP}$ violation (e.g., in neutrino oscillations). We recall that leptogenesis [39] is a simple mechanism which allows to explain the observed baryon asymmetry of the Universe [40], namely the observed difference in the present epoch of the evolution of the Universe of the number densities of baryons and anti-baryons, $n_{\mathrm{B}}$ and $n_{\overline{\mathrm{B}}}$ :

$$
Y_{\mathrm{B}}^{C M B}=\frac{n_{B}-n_{\bar{B}}}{n_{\gamma 0}}=(6.10 \pm 0.04) \times 10^{-10},
$$

where $n_{\gamma 0}$ is the photon number density in the current epoch ${ }^{3}$. The simplest scheme in which the leptogenesis mechanism can be implemented is the type I see-saw model. In its minimal version it includes the Standard Theory plus two or three heavy (RH) Majorana neutrinos, $N_{k}$. Thermal leptogenesis (see, e.g., ref. [41]) can take place, e.g., in the case of hierarchical spectrum of the

\footnotetext{
${ }^{3}$ Some times (see, e.g., $\left.[31,32,35]\right)$ the difference between number densities of baryons and anti-baryons, $\left(n_{\mathrm{B}}-n_{\overline{\mathrm{B}}}\right)$, is normalised in the expression for $Y_{\mathrm{B}}$ to the entropy density at the current epoch $s_{0}$. The entropy density $s$ at temperature $T$ is given by $s=g_{*}\left(2 \pi^{2} / 45\right) T^{3}$, where $g_{*}$ is the number of (thermalised) degree of freedom at temperature $T$. In the present epoch of the evolution of the Universe we have $s_{0}=7.04 n_{\gamma 0}$.
} 
heavy neutrino masses, $M_{1} \ll M_{2} \ll M_{3}$, which we consider in what follows. The lepton asymmetry is produced in the Early Universe in out-of-equilibrium lepton number and CP nonconserving decays of the lightest heavy Majorana neutrino, $N_{1}$, mediated by the neutrino Yukawa couplings, $\lambda$. The lepton asymmetry is converted into a baryon asymmetry by $(B-L)$-conserving but $(B+L)$ violating sphaleron interactions [42] which exist within the Standard Theory and are efficient at temperatures $100 \mathrm{GeV} \lesssim T \lesssim 10^{12} \mathrm{GeV}$. In grand unified theories the heavy neutrino masses fall typically in the range of $\sim\left(10^{8}-10^{14}\right) \mathrm{GeV}$ (see, e.g., ref. [38]). This range coincides with the range of values of $M_{k}$, required for a successful thermal leptogenesis [41]. For hierarchical spectrum of the heavy neutrino masses $M_{1} \ll M_{2} \ll M_{3}$, leptogenesis takes place in the Early Universe typically at temperatures somewhat smaller than the mass of $N_{1}$, but not smaller than roughly $10^{9}$ $\mathrm{GeV}, 10^{9} \mathrm{GeV} \lesssim T<M_{1}$. If the heavy neutrino mass spectrum is not hierarchical but not quasidegenerate either, $M_{3} \cong 3 M_{2} \cong 9 M_{1}$, successful leptogenesis can take place for values of $M_{1}$ as low as $10^{6} \mathrm{GeV}[33,34]$.

In our further discussion it is convenient to use the "orthogonal parametrisation" of the matrix of neutrino Yukawa couplings [43]:

$$
\lambda=v^{-1} \sqrt{M} R \sqrt{m} U^{\dagger}, R R^{T}=R^{T} R=\mathbf{1},
$$

where $R$ is, in general, a complex matrix. It is parametrised, in general, by six real parameters (e.g., three complex angles), of which three parameters can have CP violating values. We will use the following parametrisation of the matrix $R$ :

$$
R=\left(\begin{array}{ccc}
1 & 0 & 0 \\
0 & c_{1} & s_{1} \\
0 & -s_{1} & c_{1}
\end{array}\right)\left(\begin{array}{ccc}
c_{2} & 0 & s_{2} \\
0 & 1 & 0 \\
-s_{2} & 0 & c_{2}
\end{array}\right)\left(\begin{array}{ccc}
c_{3} & s_{3} & 0 \\
-s_{3} & c_{3} & 0 \\
0 & 0 & 1
\end{array}\right),
$$

where $c_{i}=\cos w_{i}, s_{i}=\sin w_{i}$ and the complex angles are given by $w_{i}=x_{i}+i y_{i}, i=1,2,3$.

In the setting we are considering the only source of $\mathrm{CP}$ violation in the lepton sector is the matrix of neutrino Yukawa couplings $\lambda$. It is clear from eq. (2.4) that the CP violating parameters in the matrix $\lambda$ can have their origin from the CP violating phases in the PMNS matrix $U$, or from the $\mathrm{CP}$ violating parameters present in the matrix $R$, or else from both the $\mathrm{CP}$ violating parameters in $U$ and in $R$.

For determining the conditions under which the CP-violation responsible for leptogenesis is due exclusively to the Dirac and/or Majorana CPV phases in the PMNS matrix, it is useful to analyze the constraints which the requirement of CP-invariance imposes on the Yukawa couplings $\lambda_{j l}$, on the PMNS matrix $U$ and on the matrix $R$. These constraints read (in a certain well specified and rather widely used convention) [31]:

$$
\begin{gathered}
\lambda_{j l}^{*}=\lambda_{j l} \rho_{j}^{N}, \quad \rho_{j}^{N}= \pm 1, \quad j=1,2,3, l=e, \mu, \tau, \\
U_{l j}^{*}=U_{l j} \rho_{j}^{v}, \rho_{j}^{v}= \pm 1, \quad j=1,2,3, l=e, \mu, \tau, \\
R_{j k}^{*}=R_{j k} \rho_{j}^{N} \rho_{k}^{v}, \quad j, k=1,2,3,
\end{gathered}
$$

where $i \rho_{j}^{N}= \pm i$ and $i \rho_{k}^{v}= \pm i$ are the CP-parities of the heavy and light Majorana neutrinos $N_{j}$ and $v_{k}$ (see, e.g., refs. $[29,7]$ ). Obviously, the last would be a condition of reality of the matrix 
$R$ only if $\rho_{j}^{N} \rho_{k}^{v}=1$ for any $j, k=1,2,3$. However, we can also have $\rho_{j}^{N} \rho_{k}^{v}=-1$ for some $j$ and $k$ and in that case $R_{j k}$ will be purely imaginary. Of interest for our further analysis is, in particular, the product

$$
P_{j k m l} \equiv R_{j k} R_{j m} U_{l k}^{*} U_{l m}, k \neq m .
$$

If CP-invariance holds, we find from the conditions given above that $P_{j k m l}$ has to be real [31]:

$$
P_{j k m l}^{*}=P_{j k m l}\left(\rho_{j}^{N}\right)^{2}\left(\rho_{k}^{v}\right)^{2}\left(\rho_{m}^{v}\right)^{2}=P_{j k m l} .
$$

Consider the case when CP-invariance conditions for the PMNS matrix are satisfied and $U_{\tau k}^{*} U_{\tau m}$ for given $k$ and $m, k<m, k=1,2, m=2,3$, is purely imaginary, i.e., $\operatorname{Re}\left(U_{\tau k}^{*} U_{\tau m}\right)=0$. This can be realised for $\delta=\pi q, q=0,1,2$, and $\rho_{k}^{v} \rho_{m}^{v}=-1$, i.e., if the relative CP-parity of the light Majorana neutrinos $v_{k}$ and $v_{m}$ is equal to $(-1)$, or, correspondingly, if $\alpha_{m k}=\pi\left(2 q^{\prime}+1\right), q^{\prime}=0,1, \ldots$ In this case CP-invariance holds in the lepton sector at "low" energies. In order for CP-invariance to hold at "high" energy, i.e., for $P_{j k m l}$ to be real, the product $R_{j k} R_{j m}$ has also to be purely imaginary, $\operatorname{Re}\left(R_{j k} R_{j m}\right)=0$. Thus, in the case considered, purely imaginary $U_{\tau k}^{*} U_{\tau m} \neq 0$ and real $R_{j k} R_{j m} \neq 0$, i.e., $\operatorname{Re}\left(U_{\tau k}^{*} U_{\tau m}\right)=0, \operatorname{Im}\left(R_{j k} R_{j m}\right)=0$, in particular, imply violation of CP-symmetry at "high" energy by the interplay of the matrices $U$ and $R$.

The realization that the $\mathrm{CP}$ violation necessary for the generation of the baryon asymmetry of the Universe can be due exclusively to the CPV phases in the PMNS matrix, is related to the progress in the understanding of the importance of lepton flavour effects in leptogenesis [44, 45] (for earlier discussion see ref. [46]). In the case of hierarchical heavy neutrinos $N_{k}, M_{1} \ll M_{2} \ll M_{3}$, the flavour effects in leptogenesis can be significant for $[44,45] 10^{9} \mathrm{GeV} \lesssim M_{1} \lesssim(0.5-1.0) \times 10^{12}$ $\mathrm{GeV}$. For heavy neutrinos with a mass spectrum which is not hierarchical or quasi-degenerate, $M_{3} \cong 3 M_{2} \cong 9 M_{1}$, the flavour effects in leptogenesis were shown in [33, 34] to be significant for $M_{1} \gtrsim 10^{6} \mathrm{GeV}$. It was also shown recently [34] that actually the flavour effects persist up to a very high scale, $M_{1} \gg 10^{12} \mathrm{GeV}$, i.e., beyond the usually assumed maximal scale of $M_{1}=10^{12}$ $\mathrm{GeV}[44,45]$. If the requisite lepton asymmetry is produced in one of the indicated three regimes, including the one corresponding to $10^{12} \mathrm{GeV} \leq M_{1} \lesssim 10^{13} \mathrm{GeV}, M_{1} \ll M_{2} \ll M_{3}$, the CP violation necessary for successful leptogenesis can be provided entirely by the CPV phases in the neutrino mixing matrix $[31,32,34]$.

We will consider in what follows the case when the mass of the lightest heavy neutrino $N_{1}$ lies in the interval $10^{9} \mathrm{GeV} \lesssim M_{1} \lesssim 10^{12} \mathrm{GeV}$ and the heavy Majorana neutrino mass spectrum is hierarchical, $M_{1} \ll M_{2} \ll M_{3}$. The CP violation necessary for the generation of the baryon asymmetry $Y_{B}$ in "flavoured" leptogenesis can arise, as we have already noted, both from the "low energy" neutrino mixing matrix $U$ and/or from the "high energy" part of the matrix of neutrino Yukawa couplings $\lambda$ - the matrix $R$, which can mediate CP violating phenomena only at some high energy scale determined by the masses $M_{k}$ of the heavy Majorana neutrinos $N_{k}$. The matrix $R$ does not affect the "low" energy neutrino mixing phenomenology. Suppose further that the matrix $R$ has real and/or purely imaginary CP-conserving elements: we are interested in the case when the $\mathrm{CP}$ violation necessary for leptogenesis is due exclusively to the CPV phases in $U$. Under these assumptions, $Y_{B}$ generated via leptogenesis can be written as [44, 45]:

$$
\left|Y_{B}\right| \cong 3 \times 7.04 \times 10^{-3}\left|\varepsilon_{\tau} \eta\right|
$$




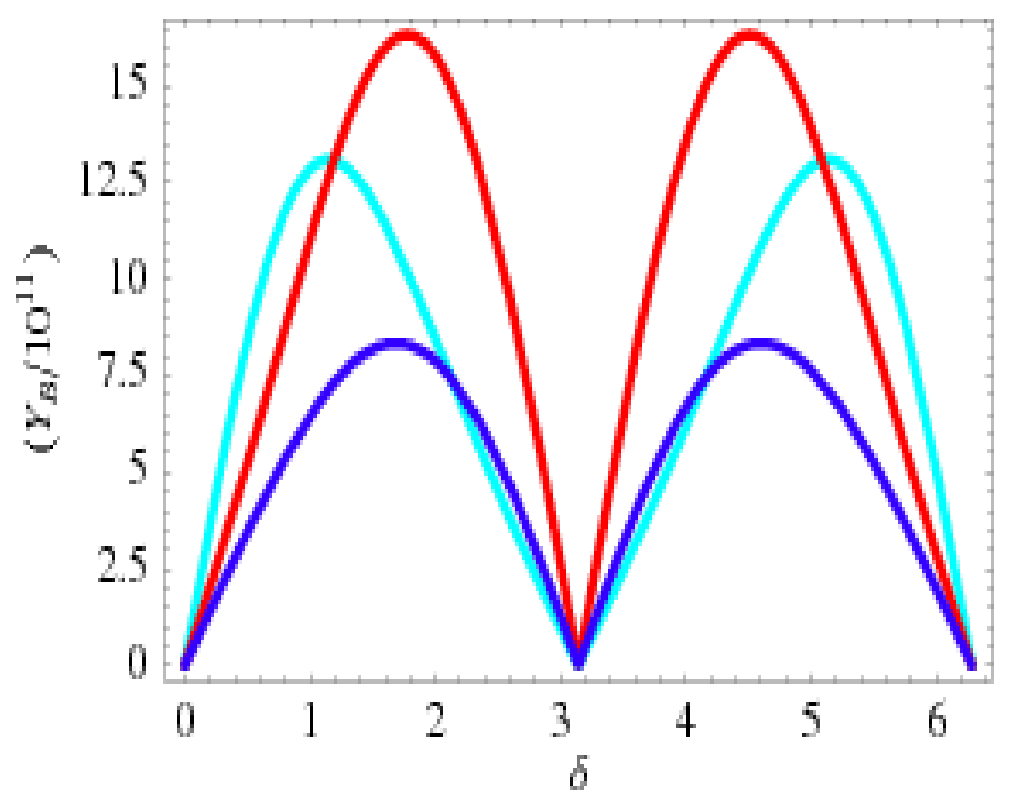

Figure 1: The baryon asymmetry $\left|Y_{B}\right|$ normalised to the entropy density $s_{0}$ as a function of the Dirac phase $\delta$ varying in the interval $\delta=[0,2 \pi]$ in the case of Dirac CP-violation, $\alpha_{32}=0 ; 2 \pi$, hierarchical heavy neutrinos and NH light neutrino mass spectrum, for $M_{1}=5 \times 10^{11} \mathrm{GeV}$, real $R_{12}$ and $R_{13}$ satisfying $\left|R_{12}\right|^{2}+\left|R_{13}\right|^{2}=1$, $\left|R_{12}\right|=0.86,\left|R_{13}\right|=0.51, \operatorname{sign}\left(R_{12} R_{13}\right)=+1$, and for i) $\alpha_{32}=0, s_{13}=0.2$ (red line) and $s_{13}=0.1$ (dark blue line), ii) $\alpha_{32}=2 \pi, s_{13}=0.2$ (light blue line). To obtain $\left|Y_{B}\right|$ normalised to the photon density $n_{\gamma 0}$ the curves must be scaled up by the factor 7.04. (The figure is from ref. [31].)

where $\varepsilon_{\tau}$ is the CPV asymmetry in the $\tau$ flavour (lepton charge) produced in $N_{1}$-decays ${ }^{4}$,

$$
\varepsilon_{\tau}=-\frac{3 M_{1}}{16 \pi v^{2}} \frac{\operatorname{Im}\left(\sum_{j k} m_{j}^{1 / 2} m_{k}^{3 / 2} U_{\tau j}^{*} U_{\tau k} R_{1 j} R_{1 k}\right)}{\sum_{i} m_{i}\left|R_{1 i}\right|^{2}},
$$

$\eta$ is the efficiency factor [44],

$$
|\eta| \cong\left|\eta\left(0.71 \widetilde{m_{2}}\right)-\eta\left(0.66 \widetilde{m_{\tau}}\right)\right|,
$$

$\widetilde{m_{2, \tau}}$ being the wash-out mass parameters which determine the rate of the processes in the Early Universe that tend to "erase", or "wash-out", the asymmetry,

$$
\widetilde{m_{2}}=\widetilde{m_{e}}+\widetilde{m_{\mu}}, \widetilde{m_{l}}=\left|\sum_{j} m_{j} R_{1 j} U_{l j}^{*}\right|^{2}, l=e, \mu, \tau
$$

Approximate analytic expression for $\eta(\widetilde{m})$ is given in $[44,45]$. We shall consider next one specific example based on the results obtained in ref. [31]. After that we shall present results found in the recent and more sophisticated analysis performed in [34].

\footnotetext{
${ }^{4}$ We have given the expression for $Y_{B}$ normalised to the photon number density $n_{\gamma 0}$.
} 
A. NH spectrum, $m_{1} \ll m_{2} \ll m_{3}, m_{2} \cong\left(\Delta m_{21}^{2}\right)^{\frac{1}{2}}, m_{3} \cong\left(\Delta m_{31}^{2}\right)^{\frac{1}{2}}$ and $N_{3}$ decoupled.

Assume for simplicity that $m_{1}$ is negligible, $m_{1} \cong 0$, and $R_{11} \cong 0$ ( $N_{3}$ decoupling). If $R_{12} R_{13}$ is real and $\alpha_{32}=0$, the only source of CP violation is the Dirac phase $\delta$ in $U$, and $\varepsilon_{\tau} \propto \sin \theta_{13} \sin \delta$. For $R_{12} R_{13}<0$ and $\left|R_{12}\right|^{2} \cong 0.85,\left|R_{13}\right|^{2}=\left(1-\left|R_{12}\right|^{2}\right) \cong 0.15$ (which maximise $\left|Y_{B}\right|$ ), we have [31]:

$$
\left|Y_{B}\right| \cong 1.04 \times 10^{-11} \frac{\left(\Delta m_{31}^{2}\right)^{\frac{1}{2}}}{0.05 \mathrm{eV}} \frac{M_{1}}{10^{9} \mathrm{GeV}} \frac{\cos ^{2} \theta_{23}}{0.449} \frac{\sin \theta_{12}}{\sqrt{0.304}} \sin \theta_{13}|\sin \delta|
$$

where ${ }^{5}$ we have used the best fit value of $\Delta m_{21}^{2}=7.34 \times 10^{-5} \mathrm{eV}^{2}$ and the efficiency factor $|\eta| \cong\left|\eta\left(0.71 \widetilde{m_{2}}\right)-\eta\left(0.66 \widetilde{m_{\tau}}\right)\right| \cong 0.043$, calculated for the best fit values of $\Delta m_{21}^{2}, \Delta m_{31}^{2}, \sin ^{2} \theta_{23}$ and $\sin ^{2} \theta_{12}$ from Table 1 (for details of the calculation of $|\eta|$ see [31]). Setting $\Delta m_{31}^{2}, \cos ^{2} \theta_{23}$ and $\sin \theta_{12}$ to their current best fit values we find that for $M_{1} \lesssim 5 \times 10^{11} \mathrm{GeV}$, for which the flavour effects in leptogenesis can be significant ${ }^{6}$, the observed value of the baryon asymmetry, taken conservatively to lie in the interval $\left|Y_{B}\right| \cong(5.90-6.30) \times 10^{-10}$, can be reproduced if

$$
\left|\sin \theta_{13} \sin \delta\right| \gtrsim 0.11 \text {. }
$$

This lower limit coincides with that found in 2006 in [31] ${ }^{7}$. A somewhat less stringent lower limit was obtained in [31] for $R_{12} R_{13}>0$ : $\left|\sin \theta_{13} \sin \delta\right| \gtrsim 0.09$ (see Fig. 1). The ranges of values of $\sin \theta_{13}|\sin \delta|$ determined by the inequalities given above are comfortably compatible with the measured value of $\sin \theta_{13} \cong 0.15$ and with the hints that $\delta \cong 3 \pi / 2$.

As was shown in [31], we can have successful leptogenesis in the discussed case also if the sole source of CP-violation is the difference of the Majorana phases $\alpha_{32}=\alpha_{31}-\alpha_{21}$ of $U_{\mathrm{PMNS}}$. In this case values of $M_{1} \gtrsim 4 \times 10^{10} \mathrm{GeV}$ are required.

B. $10^{9}<M_{1}<10^{12} \mathrm{GeV}, M_{3}>3 M_{2}>9 M_{1}$, NO Spectrum, $m_{1}<m_{2}<m_{3}$.

A rather comprehensive analysis of the possibility that the $\mathrm{CP}$ violation in leptogenesis is provided exclusively by the CPV phases in the PMNS matrix was performed recently in [34]. The baryon asymmetry was calculated employing the density matrix formalism which allows to account better for quantum correlations in the generation of the asymmetry than the Boltzmann equation approach. Results were presented, in particular, for a specific form of the $R$-matrix for which a successful (three-flavour) leptogenesis can take place at values of the lightest heavy neutrino mass $M_{1}$ as low as $10^{6} \mathrm{GeV}^{8}$. As was shown in [34] working with the parametrisation given in eq. (2.5), the elements of the $R$ matrix in this case can be made real or purely imaginary, and thus satisfying

\footnotetext{
${ }^{5}$ Here we are updating the results derived in [31] by taking into account the current best fit values of the neutrino oscillation parameters and the latest Planck data on $Y_{B}$. We are using also the normalisation of the baryon asymmetry to the photon number density $n_{\gamma 0}$, while in $[31]$ (and $[32,35]$ ) the normalisation to the entropy density $s_{0}$ has been used.

${ }^{6}$ The behavior of $Y_{B}$ in the case of real $R_{12}$ and $R_{13}$ as $M_{1}$ increases beyond $5 \times 10^{11} \mathrm{GeV}$ is rather complicated: $Y_{B}>0$ sharply decreases first, possibly goes through zero at $M_{1} \cong 10^{12} \mathrm{GeV}$ and changes sign [34].

${ }^{7}$ In [31] the following values of the oscillation parameters have been used: $\Delta m_{21}^{2}=8.0 \times 10^{-5} \mathrm{eV}^{2}, \Delta m_{31}^{2}=$ $2.5 \times 10^{-3} \mathrm{eV}^{2}, \sin ^{2} \theta_{23}=0.5$ and $\sin ^{2} \theta_{23}=0.31$. For these values $\left|Y_{B}\right|$ is maximised also by $\left|R_{12}\right|^{2} \cong 0.85,\left|R_{13}\right|^{2}=$ $\left(1-\left|R_{12}\right|^{2}\right) \cong 0.15$.

${ }^{8}$ This requires, in particular, that the heavy neutrino mass spectrum is not hierarchical (and is not quasi-degenerate either), $M_{3} \cong 3 M_{2} \cong 9 M_{1}$.
} 

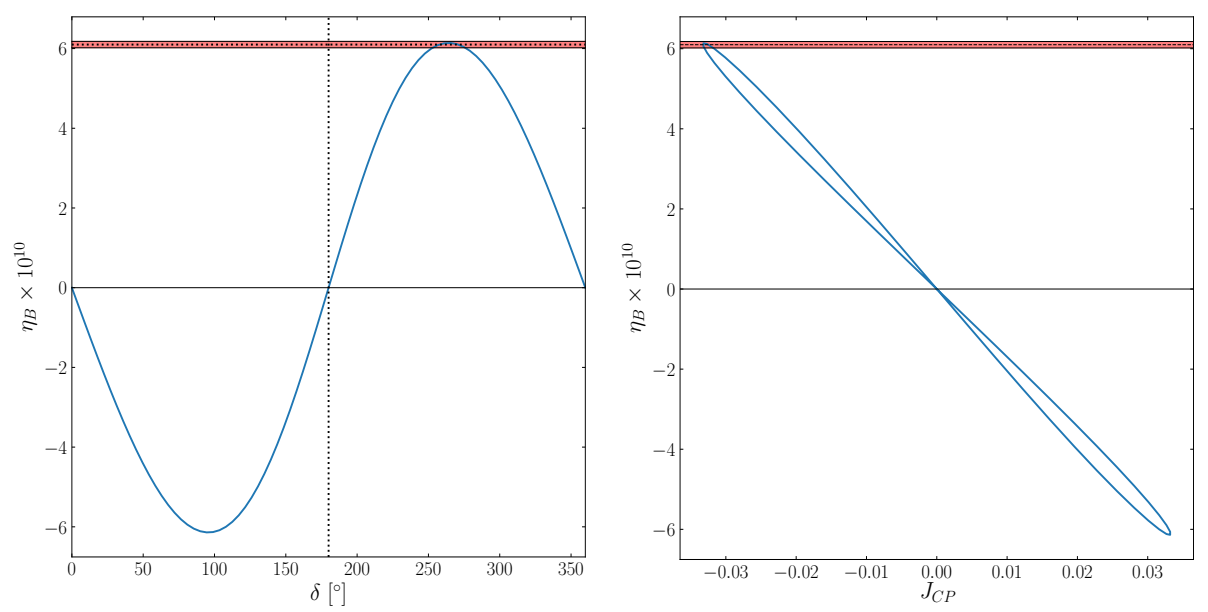

Figure 2: The baryon asymmetry $Y_{B}\left(\eta_{B} \equiv Y_{B}\right)$ with $M_{1}=5.13 \times 10^{10} \mathrm{GeV}$ and CP violation provided solely by $\delta$. The Majorana phases are fixed at $\alpha_{21}=180^{\circ}$ and $\alpha_{31}=0^{\circ}$. The red band indicates the $1 \sigma$ observed values for $Y_{B}^{C M B}$ with the best-fit value indicated by the horizontal black dotted line. Left: The final baryon asymmetry as a function of $\delta$ with exact $\mathrm{CP}$ invariance when $\delta=0^{\circ}$ and $180^{\circ}$ (vertical black dotted line). Right: A parametric plot of $\eta_{B}\left(\equiv Y_{B}\right)$ against $J_{C P}$ as $\delta$ is varied. (The figure is from ref. [34].)

the CP invariance constraints, e.g., by the following choices:

$$
x_{1}=90^{\circ}, x_{3}=180^{\circ}, y_{2}=0 .
$$

Choosing the lightest neutrino mass and the remaining $R$-matrix parameters to have the "bench mark" values,

$$
m_{1}=0.02 \mathrm{eV}, y_{1}=y_{3}=-33^{\circ}, x_{2}=18^{\circ},
$$

it was shown in that one can have a successful (two-flavour) leptogenesis when the requisite $\mathrm{CP}$ violation is provided by a) the Dirac phase $\delta$, b) by the Majorana phase $\alpha_{21}$, or c) by the Majorana phase $\alpha_{31}$ of the PMNS matrix.

\section{a) Dirac Phase CP Violation}

Given the chosen pattern of $R$-matrix angles, eqs. (2.17) and (2.18), setting $\alpha_{21}=180^{\circ}$ and $\alpha_{31}=0^{\circ}$ ensures that any CP violation in leptogenesis comes solely from $\delta$. Performing a detailed numerical parameter exploration it was found [34] that purely Dirac phase $C P$ violation leads to successful leptogenesis for $M_{1}=5.13 \times 10^{10} \mathrm{GeV}, M_{2}=2.19 \times 10^{12} \mathrm{GeV}$ and $M_{3}=1.01 \times 10^{13}$ $\mathrm{GeV}^{9}$. This result is illustrated in Fig. 2 in which the plotted $\eta_{B} \equiv Y_{B}$ comes from solving the full density matrix equations. Since both $Y_{B}$ and $J_{\mathrm{CP}}$ depend on $\delta$, for given values of the other relevant parameters there exists a correlation between the values of $Y_{B}$ and $J_{\mathrm{CP}}$. This correlation is illustrated in Fig. 2 as well.

b) CP Violation from the Majorana Phase $\alpha_{21}$

\footnotetext{
${ }^{9}$ The quoted value of $M_{1}$ is the minimal one in the considered case for which successful leptogenesis is possible with the requisite CP violation provided only by the Dirac CPV phases $\delta$.
} 


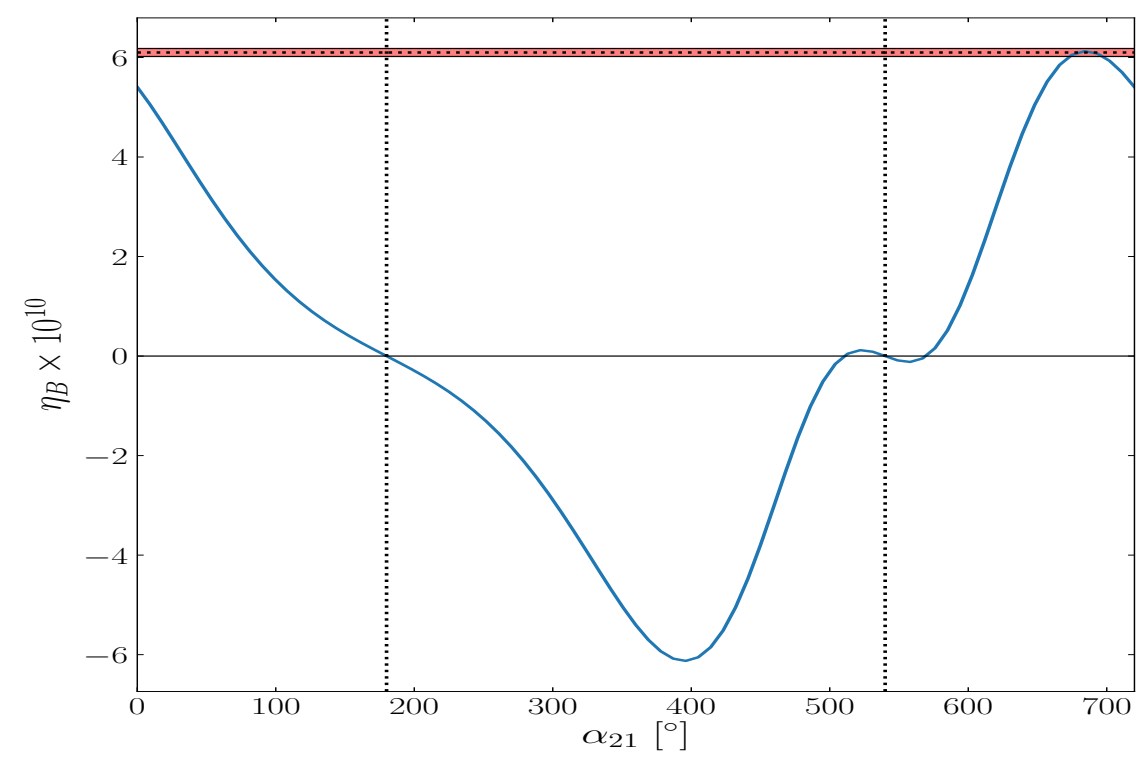

Figure 3: The baryon asymmetry $Y_{B}\left(\eta_{B} \equiv Y_{B}\right)$ with $M_{1}=3.05 \times 10^{10} \mathrm{GeV}$ and CP violation provided solely by $\alpha_{21}\left(\delta=\alpha_{31}=0^{\circ}\right)$. The red band indicates the $1 \sigma$ observed values for $Y_{B}^{C M B}$ with the best-fit value indicated by the horizontal black dotted line. The baryon asymmetry is shown against $\alpha_{21}$ with exact CP invariance at $\alpha_{21}=180^{\circ}$ and $540^{\circ}$ (vertical black dotted lines). (The figure is from ref. [34].)

In this case the phases $\delta$ and $\alpha_{31}$ are set to $\delta=\alpha_{31}=0^{\circ}$. The CP violation is generated by the Majorana phase $\alpha_{21}$. Setting all other parameters to their benchmark values, eqs. (2.17) and (2.18), and $M_{2}=10^{13} \mathrm{GeV}, M_{3}=3.16 \times 10^{13} \mathrm{GeV}, M_{1} \ll M_{2,3}$, one finds [34]:

$$
\varepsilon_{\tau}=3.14 \times 10^{-7} \cos \frac{\alpha_{21}}{2} \text {. }
$$

It follows from this expression for $\varepsilon_{\tau}$ that at the CP-conserving values for $\alpha_{21}=0^{\circ}, 360^{\circ}$ we have $\varepsilon_{\tau} \neq 0$ (see also Fig. 3). This corresponds to the case of CP-conserving $R$-matrix, CP-conserving PMNS matrix, but CP-violating interplay between the $R$ and PMNS matrix elements in leptogenesis [32]. The minimal value of $M_{1}$ which allows for a successful leptogenesis is found to be $M_{1}=3.05 \times 10^{10} \mathrm{GeV}$. The results obtained for this value of $M_{1}$ are plotted in Fig. 3. The deviation from pure (co)sinusoidal behavior is explained by the $\alpha_{21}$-dependence of the efficiency factors. The extra zeros of $\eta_{B}\left(\equiv Y_{B}\right)$ that are seen in Fig. 3 are due only to accidental cancellations and do not correspond to cases of CP symmetry (see ref. [34] for further details).

\section{b) CP Violation from the Majorana Phase $\alpha_{31}$}

Now $\delta$ and $\alpha_{21}$ are set to $\delta=0^{\circ}$ and $\alpha_{21}=180^{\circ}$ such that $\mathrm{CP}$ violation is provided by $\alpha_{31}$. Using the benchmark values for the other parameters from eqs. (2.17) and (2.18) and $M_{2}=10^{13}$ $\mathrm{GeV}, M_{3}=3.16 \times 10^{13} \mathrm{GeV}, M_{1} \ll M_{2,3}$, one gets [34]:

$$
\varepsilon_{\tau}=2.11 \times 10^{-7} \sin \frac{\alpha_{31}}{2} .
$$

The minimal value of $M_{1}$ at which the observed baryon asymmetry is created is found to be $M_{1}=$ $5.13 \times 10^{10} \mathrm{GeV}$. The dependence of $Y_{B}$ on $\alpha_{31}$ is plotted in Fig. 4. The analytic expectation of a 


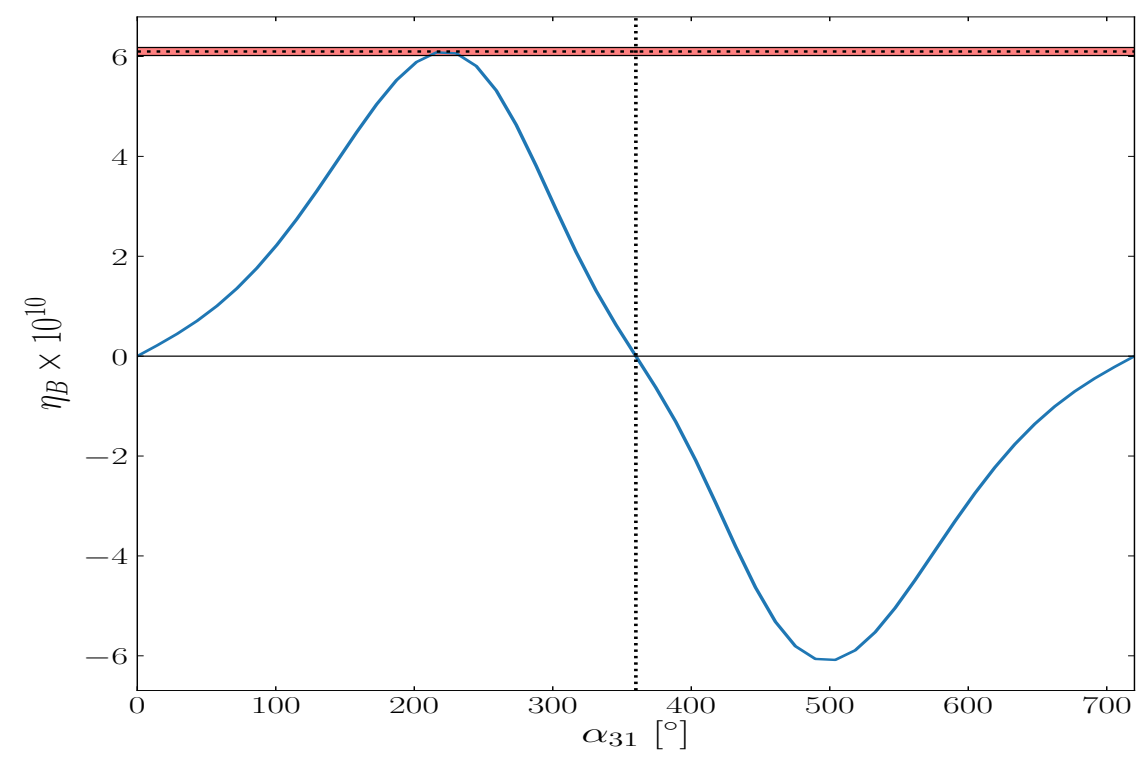

Figure 4: The baryon asymmetry $\eta_{B}\left(\equiv Y_{B}\right)$ with $M_{1}=5.13 \times 10^{10} \mathrm{GeV}$ and $C P$ violation provided solely by $\alpha_{31}$ (corresponding to $\delta=0$ and $\alpha_{21}=180^{\circ}$ ). The red band indicates the $1 \sigma$ observed values for $Y_{B}^{C M B}$ with the best-fit value indicated by the horizontal black dotted line. The baryon asymmetry is shown against $\alpha_{31}$ with exact CP invariance taking place $\alpha_{31}=0^{\circ}$ and $360^{\circ}$ (vertical black dotted lines). (The figure is from ref. [34].)

sinusoidal dependence of the baryon asymmetry on $\alpha_{31} / 2\left(Y_{B} \propto \varepsilon_{\tau} \propto \sin \left(\alpha_{31} / 2\right)\right)$ from eq. (2.20) is clearly present in Fig. 4 (with a minor distortion due to the efficiency factor). It follows from the expression for $\varepsilon_{\tau}$, eq. (2.20), and Fig. 4, that at the CP-conserving values of $\alpha_{31}=180^{\circ}, 540^{\circ}$ we have $\varepsilon_{\tau} \neq 0$ and $Y_{B} \neq 0$. This again corresponds to the case of CP-conserving $R$-matrix, CPconserving PMNS matrix, but CP-violating interplay between the $R$ and PMNS matrix elements in leptogenesis [32].

\section{Outlook}

The program of research in neutrino physics aims at shedding light on some of the fundamental aspects of neutrino mixing:

i) the status of CP symmetry in the lepton sector;

ii) the nature of massive neutrinos $v_{j}$, which can be Dirac fermions possessing distinct antiparticles, or Majorana fermions, i.e., spin 1/2 particles that are identical with their antiparticles;

iii) the type of spectrum the neutrino masses obey;

iv) the absolute scale of neutrino masses. 
The program extends beyond the year 2030 (see, e.g., refs. [5, 48, 49, 50, 47, 51, 52, 53, $54,55])$. Our ultimate goal is to understand at a fundamental level the mechanism giving rise to neutrino masses and mixing and to non-conservation of the lepton charges $L_{l}, l=e, \mu, \tau$. This includes understanding the origin of the patterns of neutrino mixing and of neutrino masses suggested by the data. The remarkable experimental program of research in neutrino physics and the related theoretical efforts are stimulated by the fact that the existence of nonzero neutrino masses and the smallness of the neutrino masses suggest the existence of new fundamental mass scale in particle physics, i.e., the existence of New Physics beyond that predicted by the Standard Theory. It is hoped that progress in the theory of neutrino mixing will also lead, in particular, to progress in the theory of flavour and to a better understanding of the mechanism of generation of the baryon asymmetry of the Universe.

Acknowledgements. I would like to thank P. Petroff and his colleagues for organising such a scientifically enjoyable Conference. This work was supported in part by the INFN program on Theoretical Astroparticle Physics (TASP), by the European Union Horizon 2020 research and innovation programme under the Marie Sklodowska-Curie grants 674896 and 690575, and by the World Premier International Research Center Initiative (WPI Initiative, MEXT), Japan.

\section{References}

[1] B. Pontecorvo, Zh. Eksp. Teor. Fiz. 33 (1957) 549 and 34 (1958) 247.

[2] Z. Maki, M. Nakagawa and S. Sakata, Prog. Theor. Phys. 28 (1962) 870.

[3] B. Pontecorvo, Zh. Eksp. Teor. Fiz. 53 (1967) 1717.

[4] Y. Fukuda et al. [Super-Kamiokande Collaboration], Phys. Rev. Lett. 81 (1998) 1562 [hep-ex/9807003].

[5] K. Nakamura and S.T. Petcov in M. Tanabashi et al. [Particle Data Group], Phys. Rev. D 98 (2018) 030001.

[6] S. M. Bilenky, J. Hosek and S. T. Petcov, Phys. Lett. B 94 (1980) 495.

[7] S. M. Bilenky and S. T. Petcov, Rev. Mod. Phys. 59 (1987) 671.

[8] F.P. An et al., Phys. Rev. Lett. 108 (2012) 171803.

[9] J.K. Ahn et al., Phys. Rev. Lett. 108 (2012) 191802.

[10] Y. Abe et al. [Double Chooz Collaboration], Phys. Lett. B 723 (2013) 66. Y. Abe et al.,Phys. Lett. B 735 (2014) 51

[11] F. Capozzi et al., Prog. Part. Nucl. Phys. 102 (2018) 48 [arXiv:1804.09678 [hep-ph]].

[12] I. Esteban et al., NuFIT webpage http://www.nu-fit.org (see v3.2: Three-neutrino fit based on data available in January 2018).

[13] F. Capozzi et al., Phys. Rev. D 89 (2014) 093018.

[14] J. T. Penedo and S. T. Petcov, Phys. Lett. B 786 (2018) 410.

[15] X. Qian and P. Vogel, Prog. Part. Nucl. Phys. 83 (2015) 1.

[16] S.T. Petcov, M. Piai, Phys. Lett. B 533 (2002) 94. S. Pascoli and S.T. Petcov, Phys. Lett. B 544 (2002) 239. J. Bernabéu et al., Nucl. Phys. B 669 (2003) 255; S.T. Petcov and S. Palomares-Ruiz, Nucl. Phys. B 712 (2005) 392; S.T. Petcov, T. Schwetz, Nucl. Phys. B 740 (2006) 1. 
[17] F. Perrin, Comptes Rendus 197, 868 (1933);E. Fermi, Nuovo Cim. 11 (1934) 1.

[18] Ch. Kraus et al., Eur. Phys. J. C40 (2005) 447.

[19] V. Lobashev et al., Nucl. Phys. A 719 (2003) 153c.

[20] V.N. Aseev et al., Phys. Rev. D 84 (2011) 112003.

[21] K. Eitel et al., Nucl. Phys. B (Proc. Suppl.) 143 (2005) 197.

[22] K. N. Abazajian and M. Kaplinghat, Ann. Rev. Nucl. Part. Sci. 66 (2016) 401.

[23] N. Aghanim et al., Astron. Astrophys. 596 (2016) A107 [arXiv:1605.02985].

[24] S.M. Koksbang and S. Hannestad, JCAP 1709 (2017) 014

[25] N. Cabibbo, Phys. Lett. B 72 (1978) 333.

[26] P.I. Krastev and S.T. Petcov, Phys. Lett. B 205 (1988) 84.

[27] C. Jarlskog, Z. Phys. C29 (1985) 491.

[28] P. Langacker et al., Nucl. Phys. B 282 (1987) 589.

[29] S.T. Petcov, Adv. High Energy Phys. 2013 (2013) 852987 [arXiv:1303.5819]; S.M. Bilenky, S. Pascoli and S.T. Petcov, Phys. Rev. D 64 (2001) 053010.

[30] J. D. Vergados, H. Ejiri and F. Šimkovic, Int. J. Mod. Phys. E 25 (2016) 1630007 [arXiv:1612.02924].

[31] S. Pascoli, S. T. Petcov and A. Riotto, Nucl. Phys. B 774 (2007) 1;

[32] S. Pascoli, S. T. Petcov and A. Riotto, Phys. Rev. D 75 (2007) 083511

[33] K. Moffat et al., Phys. Rev. D 98 (2018) 015036.

[34] K. Moffat et al., arXiv:1809.08251 [hep-ph].

[35] E. Molinaro, S.T. Petcov, Phys. Lett. B 671 (2009) 60; Eur. Phys. J. C 61 (2009) 93.

[36] P. Chen, G.-J. Ding and S.F. King, JHEP 1603 (2016) 206; C. Hagedorn and E. Molinaro, Nucl. Phys. B 919 (2017) 404.

[37] P. Minkowski, Phys. Lett. B 67, 421 (1977); T. Yanagida in Proc. of the Workshop on Unified Theory and Baryon Number of the Universe, KEK, Japan, 1979; M. Gell-Mann, P. Ramond and R. Slansky in Sanibel Talk, CALT-68-709, Feb 1979, and in Supergravity (North Holland, Amsterdam 1979); S.L.Glashow, Cargese Lectures (1979).

[38] R. Mohapatra et al., Rept. Prog. Phys. 70, 1757 (2007); A. Bandyopadhyay et al., Rept. Prog. Phys. 72, 106201 (2009); D. Meloni, Front. in Phys. 5 (2017) 43 [arXiv:1709.02662 [hep-ph]], and references quoted therein.

[39] M. Fukugita and T. Yanagida, Phys. Lett. B 174 (1986) 45.

[40] P.A.R. Ade et al. [Planck Collab.], Astronom. Astrophys. 594 (2016) A13 [arXiv:1502.01589 [astro-ph]].

[41] W. Buchmuller et al., Annals Phys. 315 (2005) 305.

[42] V.A. Kuzmin et al., Phys. Lett. B 155 (1985) 36.

[43] J.A. Casas and A. Ibarra, Nucl. Phys. B 618 (2001) 171.

[44] A. Abada et al., JCAP 0604 (2006) 004; A. Abada et al., JHEP 0609 (2006) 010. 
[45] E. Nardi et al., JHEP 0601 (2006) 164.

[46] R. Barbieri et al., Nucl. Phys. B 575 (2000) 61.

[47] R. Acciarri et al. [DUNE Collab.], arXiv:1512.06148, arXiv:1601.05471, arXiv:1601.02984.

[48] K. Abe et al. [T2K Collab.], PTEP 2015 (2015) 043C01 [arXiv:1409.7469 [hep-ex]]; arXiv:1609.04111 [hep-ex].

[49] M. Sanchez et al. [NOvA Collab.], talk at the XXVIII Int. Conf. on Neutrino Physics and Astrophysics, June 5-9, 2018, Heidelberg, Germany.

[50] K. Abe et al. [Hyper-Kamiokande Proto-Collab.], PTEP 2015 (2015) 053C02 [arXiv:1502.05199].

[51] S. Ahmed et al. [ICAL Collab.], Pramana 88 (2017) 79 [arXiv:1505.07380 [physics.ins-det]].

[52] F. An et al. [JUNO Collab.], J. Phys. G 43 (2016) 030401 [arXiv:1507.05613].

[53] S. Adrian-Martinez et al. [KM3Net Collab.], J. Phys. G 43 (2016) 084001 [arXiv:1601.07459 [astro-ph.IM]].

[54] K. Abe et al. [Hyper-Kamiokande Proto-Collab.], PTEP 2018 (2018) 063C01 [arXiv:1611.06118v3 [hep-ex]].

[55] E. Baussan et al. [ESSnuSB Collab.], Nucl. Phys. B 885 (2014) 127. 